\title{
Marking the operative site: a lesson learned
}

\author{
Dominique M.A. Knight MBBS, John H. Wedge OC MD
}

Previously published at www.cmaj.ca

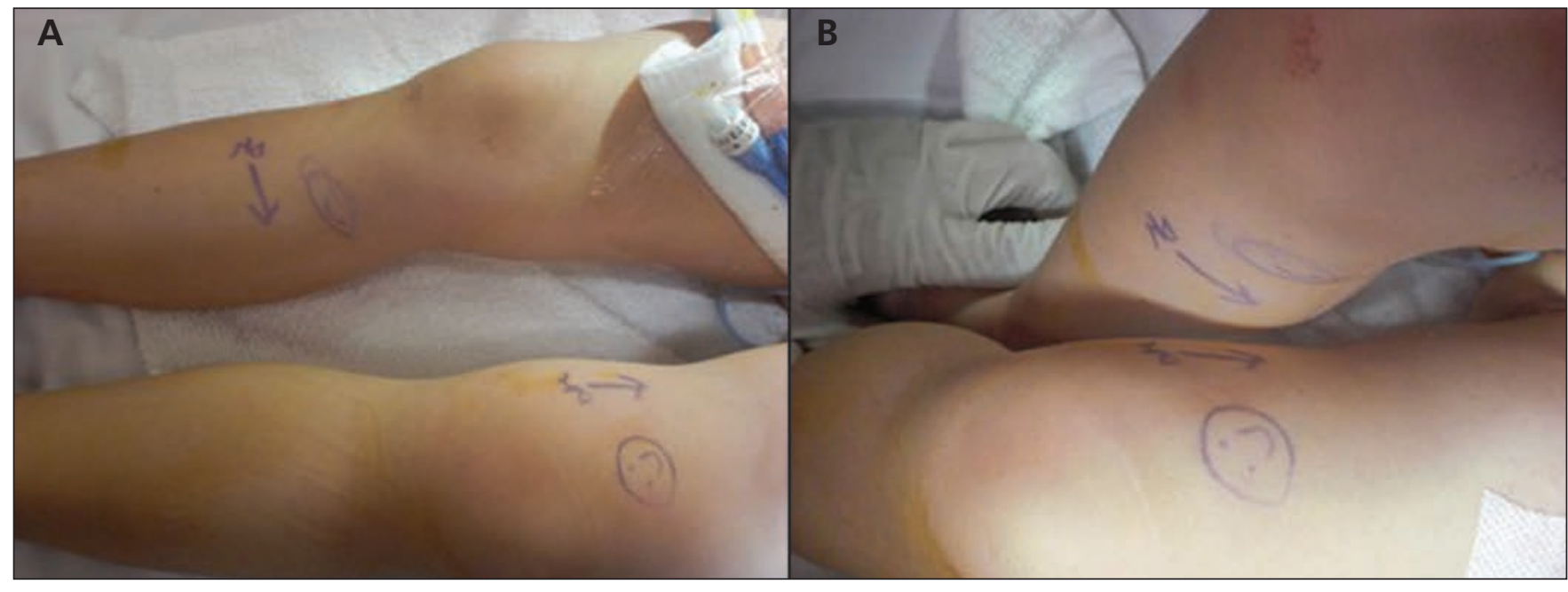

Figure 1: (A) Lower limbs of a two-year-old child with surgical marks on both legs. (B) Probable preoperative positioning of the legs with resultant transfer of the surgical mark from the left thigh to the right calf.

A two-year-old child was admitted for elective orthopedic surgery to the left proximal femur. While the child was in the preoperative area, the thigh was clearly marked. A permanent pen was used to draw an arrow and a smiling face and to write the surgeon's initials.

When the child arrived in the operating room, it appeared that both legs had been marked for surgery (Figure 1A). The operating surgeons confirmed the surgical site from the notes, parental consent form and preoperative radiographs. The image intensifier was used to verify the surgical location before the operation. Although standard protocols had been followed, the patient's resting position in the preoperative area had facilitated arrow transfer to the incorrect opposite lower leg (Figure 1B).

Surgery on the wrong side is a devastating error and hospitals have standard measures in place to prevent this; however, reports of wrong-sided surgery have occurred in even the most highly regarded institutions. ${ }^{1}$ Meinberg and Stern ${ }^{2}$ surveyed more than 1000 hand surgeons and found that $21 \%$ admitted

From the Division of Orthopaedic Surgery (Knight), Hospital for Sick Children and the Department of Surgery (Wedge), University of Toronto, Toronto, Ont.

\section{CMAJ 2010. DOI:10.1503/cmaj.091860}

operating on the wrong site, and a further $16 \%$ had found that they had prepared the wrong side just before incision.

Multiple safety measures should be implemented to avoid surgical misadventure. In our unit, the preoperative mark is performed by a surgeon who will be present during surgery. In the operating room, before surgical draping, the patient's name and limb mark are checked and the consent form with images are then reviewed.

In addition to these precautions, there should be careful consideration given to placement of surgical marks away from potential areas of transfer. In addition to an initialled arrow pointing to the surgical location, it may be helpful to clearly write the site and side. These extra measures may help to ensure that arrow transposition, in isolation, will not contribute to surgery performed on the incorrect side.

This article has been peer reviewed.

Competing interests: None declared.

\section{REFERENCES}

1. Altman LK. State issues scathing report on error at Sloan-Kettering. The New York Times 1995 Nov. 16; Sect A:1, A:16.

2. Meinberg EG, Stern PJ. Incidence of wrong-site surgery among hand surgeons. J Bone Joint Surg Am 2003;85:193-7. 